\title{
The s-process Nucleosynthesis
}

\author{
S. Goriely and L. Siess \\ Institut d'Astronomie et d'Astrophysique, ULB, Campus de la Plaine, CP226, B-1050 \\ Brussels, Belgium \\ email: sgoriely@astro.ulb.ac.be and siess@astro.ulb.ac.be
}

\begin{abstract}
Theoretical as well as observational aspects of the s-process nucleosynthesis are reviewed. The classical site-independent s-process model as well as the s-process in massive stars are shortly described. A special attention is paid to the nucleosynthesis taking place in AGB stars and the extra-mixing invoked to explain the production of neutrons in the C-rich layers during the interpulse. We also discuss the nucleosynthesis found in hot AGB stars for which the s-process during the interpulse phase is inhibited, but the one resulting from the large temperatures in the thermal pulse is boosted. We comment on the uncertainties affecting our understanding of the physical mechanisms responsible for a successful s-process. Finally, various types of spectroscopic observations of s-process elements are discussed.
\end{abstract}

Keywords. Nuclear reactions, nucleosynthesis, abundances, stars: AGB and post-AGB

\section{Introduction}

For the last decades, an extremely intense amount of work has been devoted to the slow neutron-capture process (or s-process) of nucleosynthesis called to explain the origin of about half of the elements heavier than iron observed in nature (see e.g Seeger et al. 1965; Käppeler et al. 1989). Although the astrophysical modelling of the s-process still faces some problems, our understanding of the nuclear mechanisms responsible for the production of the s-nuclei can be regarded as quite satisfactory, at least in comparison with the other important nucleosynthetic process susceptible to produce elements heavier than iron, namely the rapid neutron-capture process (or r-process). One of the major reasons for this success consists in the remarkable effort performed in experimental and theoretical nuclear physics to determine as reliably as possible the nuclear quantities of relevance to the s-process nucleosynthesis. As regards the astrophysical modelling, the situation is unfortunately less satisfactory. Although observations of the radioactive Tc in stellar envelopes clearly prove that the s-process takes place during hydrostatic nuclear burning, it remains difficult to explain the large neutron concentrations required to account for the observed abundances of s-elements. At the present time, two nuclear reactions are suggested as possible neutron sources, i.e ${ }^{13} \mathrm{C}(\alpha, \mathrm{n}){ }^{16} \mathrm{O}$ and ${ }^{22} \mathrm{Ne}(\alpha, \mathrm{n}){ }^{25} \mathrm{Mg}$. These reactions could be responsible for a large production of neutrons during core Heburning in massive stars $\left(M \gtrsim 10 M_{\odot}\right)$ (e.g Prantzos et al. 1990) and during the shell Heburning phase of thermally pulsing low and intermediate mass AGB stars $\left(M \lesssim 10 M_{\odot}\right)$ (e.g Straniero et al. 1995). Even though the core He-burning has proven its ability to produce the lightest s-elements (i.e with mass number $70 \lesssim A \lesssim 90$ ), the astrophysical models associated with AGB stars (believed to be responsible for the production of the $A>90$ s-elements) are still quite uncertain, in particular concerning the description of the mechanisms that could be at the origin of the neutron production.

In Sect. 2, the classical site-independent s-process model and its relevance are shortly introduced. In Sect. 3, the weak s-process taking place in massive stars is described, while 
Sect. 4 focuses on the s-process during the AGB phase of low- and intermediate-mass stars. Some comparisons with observations are discussed in Sect. 5.

\section{The Classical s-process Model}

Fully parametric models free of all astrophysical constraints have been introduced since the 60 s in order to estimate the ability of the slow neutron capture reactions to synthesize the various heavy elements in solar quantity. These models have shown their ability to reproduce the solar abundances of the s-only nuclei within a few tens of a percent and have since been used principally to decompose the solar abundances of heavy elements into their s-, r-, and p-components $\dagger$. The most popular of these parametric models, well-known as the classical s-process model, is based on the original canonical model of Burbidge et al. (1957) and was first successfully developed by Clayton et al. (1961). The canonical model assumes that some stellar material composed of iron nuclei only is subject to a constant neutron irradiation characterized by the fact that the neutron density is low enough for the $\beta$-decays of unstable nuclei to be faster than the neutron captures. Rapidly, it turned out that the solar system s-abundances could not be explained as originating from one given neutron exposure. The solar s-element composition was therefore assumed to be the result of a superposition of different distributions of neutron exposures. The solar s-abundance distribution clearly indicates that the stronger the neutron irradiation the less probable it becomes. In particular, Clayton et al. (1961) showed that an exponential or power law neutron exposure distribution could efficiently do the work. This model has been very successful in reproducing the solar system s-abundance distribution by considering the superposition of three exponential distributions: the weak component essentially responsible for the production of the $70 \leqslant A \leqslant 90$ s-nuclei, the main component for the $90 \leqslant A \leqslant 204$ isotopes and the strong component for the $\mathrm{Pb}$ and Bi elements (e.g Käppeler et al. 1989).

The classical s-process model has subsequently been used to derive the s-contribution to the solar system as well as the solar system r-process abundances obtained from the observed solar values by subtracting the possible s-contribution (Fig. 1). As shown in Fig. 1, large error bars still exist (for more details see Goriely 1999). These can also affect the determination of the solar r-abundances, in particular for s-dominant nuclei (Fig. 1). For example, about $80 \%$ of the solar abundance of ${ }^{140} \mathrm{Ce}$ is predicted to be produced by the classical s-process. The remaining $20 \%$ of the solar abundance are therefore ascribed to the r-process. However, a small change in the s-process parameters, such as deviations from the theoretical exponential form for the neutron exposure, could increase the scontribution, and consequently make the r-process contribution negligible. The same holds for $\mathrm{Pb}$, which can be made entirely by the s-process. However, it is well known that in principle $\mathrm{Pb}$ can also be produced in large amount by the r-process. Special attention should therefore be paid to such elements that can be produced by both the s- and r-processes. These large uncertainties in determining the s- and r-contributions as well as the mere fact that these values concern the solar system abundances only and may not be relevant to the s-process in the early age of the Galaxy should be kept in mind, especially when interpreting the observed abundances in low-metallicity stars.

$\dagger$ In addition to the neutron-capture s- and r-processes, another nucleosynthetic process (the p-process) is invoked to explain the origin of a large number of proton-rich isotopes heavier than iron which cannot be synthesized by the s- and r-processes. see Arnould \& Goriely (2003) for a review on the p-process nucleosynthesis. 

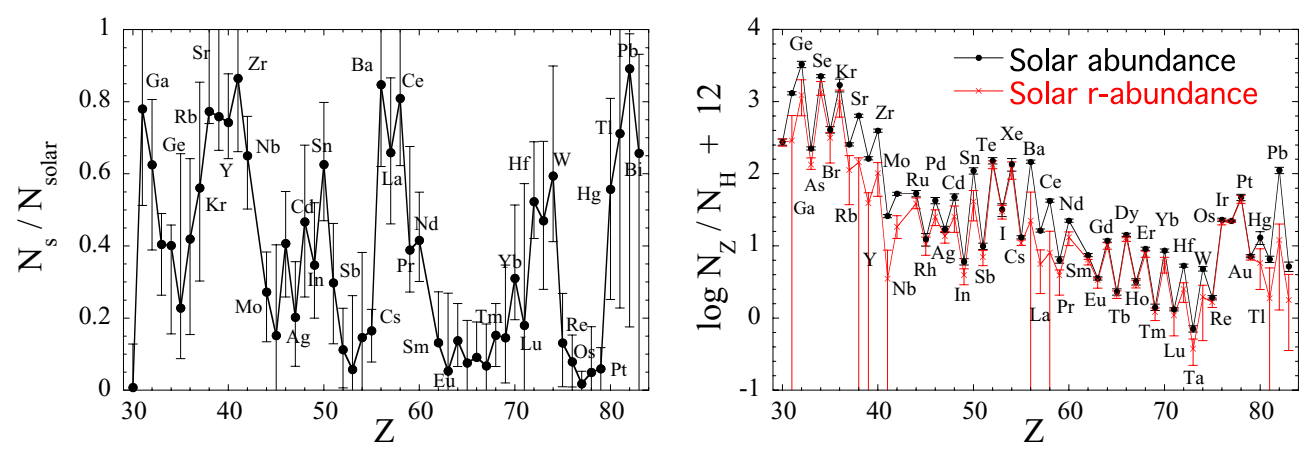

Figure 1. Left: s-process contribution to the solar system elemental abundances. The error bars correspond to the uncertainties affecting the classical model, as well as the nuclear physics input and the solar system abundances (Goriely 1999). Right: Elemental solar r-abundances (expressed in units normalized to $10^{12} \mathrm{H}$ atoms) derived from the classical model with error bars resulting from the uncertainties shown in the left panel. Also shown are the total solar system abundances.

\section{The s-process in massive stars}

It is now well accepted that the so-called weak s-process taking place during the core He-burning of massive stars can be held responsible for the galactic production of the lightest $70 \lesssim A \lesssim 90$ s-elements. In these stars, efficient convective mixing does not allow heavy elements to be subject to successive neutron irradiations. The most abundant seeds to the s-process, i.e. the iron group elements, capture most of the neutrons, and this prevents the efficient nucleosynthesis of elements beyond $A \sim 90$. At a temperature of about $T=3 \times 10^{8} \mathrm{~K},{ }^{22} \mathrm{Ne}(\alpha, \mathrm{n}){ }^{25} \mathrm{Mg}$ produces a relatively low but steady production of neutrons. The predicted s-abundance distribution is found to be relatively robust and model-independent, as shown in Fig. 2, though it remains affected by uncertainties in the description of the convective mixing and most particularly in the ${ }^{22} \mathrm{Ne}(\alpha, \mathrm{n}){ }^{25} \mathrm{Mg}$ reaction rate. The sensitivity of the s-process efficiency for sub-solar metallicity stars is discussed by Rayet \& Hashimoto (2000).

Large uncertainties in the ${ }^{22} \mathrm{Ne}(\alpha, \mathrm{n}){ }^{25} \mathrm{Mg}$ rate are reported in the NACRE compilation of reaction rates (Angulo et al. 1999) with an upper limit being about 100 times higher than the reported 'adopted' value in the 2.5 to $3.5 \times 10^{8} \mathrm{~K}$ temperature range of relevance to the s-process in massive stars (such an uncertainty has been revisited; for a discussion see Arnould \& Goriely (2003) and references therein). The distribution of s-elements in the He-burning core of a $Z=\mathrm{Z}_{\odot} 40 M_{\odot}$ model star is shown in Fig. 2 when use is made of different estimates for the ${ }^{22} \mathrm{Ne}(\alpha, \mathrm{n}){ }^{25} \mathrm{Mg}$ rate. These modifications strongly influence the production of s-elements and could, in addition, explain the still unsolved origin of the light p-process nuclei ${ }^{92,94} \mathrm{Mo}$ and ${ }^{96,98} \mathrm{Ru}$ (Arnould \& Goriely 2003) as well as the peculiar Ba overabundance observed in the SN1987A ejecta (Mazzali \& Chugai, 1995).

\section{The s-process in AGB stars}

In AGB stars, the neutrons required to explain the development of the s-process are believed to originate mainly from the ${ }^{13} \mathrm{C}(\alpha, \mathrm{n}){ }^{16} \mathrm{O}$ reaction within a thin radiative layer below the convective envelope. In low-mass AGB stars, the ${ }^{22} \mathrm{Ne}(\alpha, \mathrm{n}){ }^{25} \mathrm{Mg}$ reaction is not efficient enough for a relevant production of neutrons and is called for to explain 


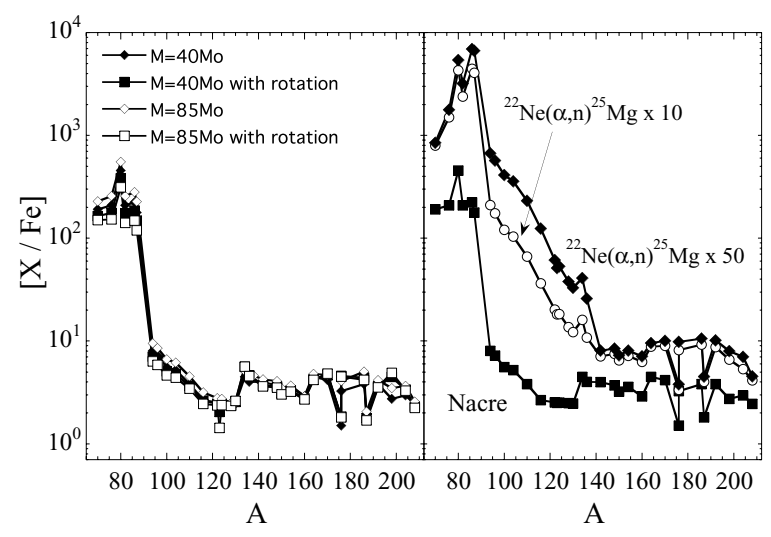

Figure 2. left panel: s-abundance overproduction factors estimated at the end of core He-burning in solar metallicity stars of different initial masses. Right panel: same as in left panel for a standard non-rotating $40 M_{\odot}$ star and different estimates of the ${ }^{22} \mathrm{Ne}(\alpha, \mathrm{n}){ }^{25} \mathrm{Mg}$ reaction rates, namely the Nacre recommended rate (Angulo et al. 1999) or 10 and 50 times the rate.

the reshaping of the s-abundance distribution for specific branching point nuclei. This s-process scenario developing in typically $1 \lesssim M\left[M_{\odot}\right] \lesssim 3$ AGB stars corresponds to the most popular model invoked to explain the observations and is described in Sect. 4.1. In contrast, there is a large lack of studies regarding the s-process in hot AGB stars, i.e massive $\left(M \gtrsim 3 M_{\odot}\right)$ and/or metal-poor stars. In this case, the temperature at the base of the convective thermal pulse can exceed $3 \times 10^{8} \mathrm{~K}$ and efficiently ignite the ${ }^{22} \mathrm{Ne}(\alpha, \mathrm{n}){ }^{25} \mathrm{Mg}$ source. Such an s-process in hot AGB stars is discussed in Sect. 4.2.

\subsection{The s-process in cold AGB stars}

In cold AGB stars, the neutrons are mainly produced by $\alpha$-captures on ${ }^{13} \mathrm{C}$ in a thin radiative layer below the convective envelope. It has long been suggested (Iben \& Renzini, 1982), that if protons from the envelope can mix partially with the carbon-rich layers left behind the pulse-driven convective shell, then ${ }^{13} \mathrm{C}$ can be synthesized through the chain of reaction ${ }^{12} \mathrm{C}(\mathrm{p}, \gamma){ }^{13} \mathrm{~N}\left(\beta^{+}\right){ }^{13} \mathrm{C}$. Although parametric studies, by artificially incorporating suitable amounts of ${ }^{13} \mathrm{C}$ into the system (Gallino et al. 1998, Busso et al. 2001) or by injecting instantaneously protons into the C-rich layers at the time of the third dredgeup (3DUP) (Goriely \& Mowlavi 2000) are able to reproduce a large set of observational heavy element abundance data, the very origin of this so-called " $13 \mathrm{C}$-pocket" is still largely unknown and remains the central problem of realistic s-process models.

The most recent attempts to identify the physical cause for this extra mixing refer to the diffusive overshoot (Herwig et al. 1997), rotationally induced mixing (Langer et al. 1999; Herwig et al. 2003; Siess et al. 2004) or gravity waves (Denissenkov \& Tout 2003). The best studied mechanism so far is the overshoot model, which predicts a fast diffusion of protons on relatively short timescales. This model leads to virtually similar predictions as the parametric approach of Goriely \& Mowlavi which consists in mixing protons into the C-rich region instantaneously or the ${ }^{13} \mathrm{C}$-pocket scenario of Gallino et al. (1998) in which a standard ${ }^{13} \mathrm{C}$ profile of protons (corresponding to an instantaneous mixing of protons) is adopted. In this framework, the s-process abundance distribution appears to be essentially a function of the stellar metallicity, as shown in Fig. 3. On the other hand, the magnitude of the surface overabundances depends on the depth of the 3DUP, the extent of the thermal pulse and the envelope mass, and consequently vary significantly with the stellar mass. 


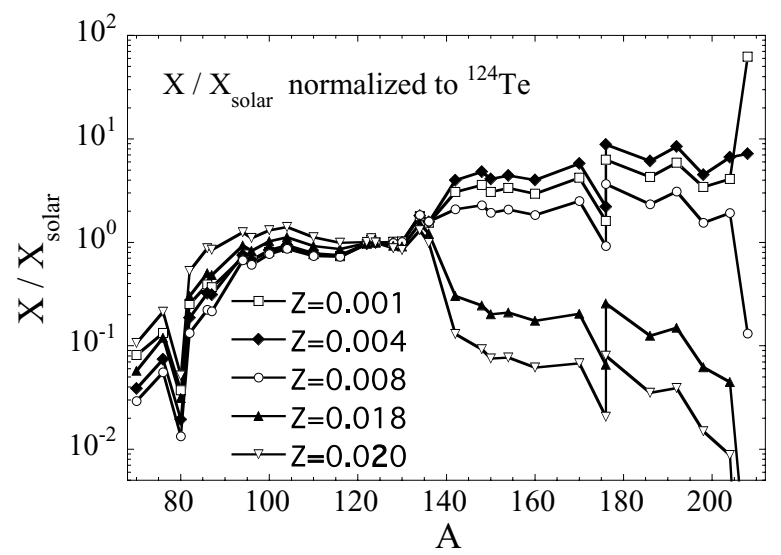

Figure 3. Abundance distribution (relative to solar) of s-only nuclei at the end of the interpulse phase for stars of different metallicities. The distribution is averaged over the mass range covered by the partial mixing region (assuming an initial exponentially decreasing $\mathrm{H}$ profile) and normalized to the solar abundance of ${ }^{124} \mathrm{Te}$. See Goriely \& Mowlavi (2000) for more details.

In these scenarios, all AGB stars, irrespective of their metallicity, are expected to produce nuclei with $90 \lesssim A \lesssim 140$ in an almost solar distribution. In contrast, only lowmetallicity stars $(Z \lesssim 0.01)$ are predicted to synthesize heavier s-nuclei $(140 \lesssim A \lesssim 204)$ efficiently. Of particular interest is also the large overproduction of $\mathrm{Pb}$ and $\mathrm{Bi}$ for stars with metallicities $Z \lesssim Z_{\odot} / 10$. More generally, the mixing scenario described above predicts that all low-metallicity AGB stars enriched in s-process elements should exhibit a large overproduction of $\mathrm{Pb}$ and $\mathrm{Bi}$ compared to other s-isotopes. Even $\mathrm{Z}=0$ stars can be included in this class of Pb-stars. Indeed, recent evolutionary models of zero-metallicity stars show that the development of mixing episodes at the beginning of the AGB phase allows low- and intermediate-mass stars to experience thermal pulses (Chieffi et al. 2001; Siess et al. 2002). If in addition these stars experience a partial mixing of protons from the H-rich envelope into the C-rich layers at the time of the 3DUP, an extensive neutron capture nucleosynthesis leads to the production of s-process nuclei with a large overabundance of $\mathrm{Pb}$ and $\mathrm{Bi}$ (Goriely \& Siess 2001).

The ashes of the radiative nucleosynthesis during the interpulse phase are ingested into the pulse-driven convective region and mixed down to the high temperatures $T^{\text {bot }}$ characterizing its base. $T^{\text {bot }}$ is known to increase with increasing pulse number, stellar mass or decreasing metallicity, and ranges from 1.5 to above $4 \times 10^{8} \mathrm{~K}$. At these high temperatures, the ${ }^{14} \mathrm{~N}$ and ${ }^{18} \mathrm{O}$ left over by the $\mathrm{H}$-burning shell during the preceding interpulse are completely transformed into ${ }^{22} \mathrm{Ne}$ by $\alpha$-capture reactions. At temperatures $T^{\text {bot }} \gtrsim 3 \times 10^{8} \mathrm{~K}$ (found in low- $Z$ and/or high- $M$ stars), ${ }^{22} \mathrm{Ne}(\alpha, \mathrm{n}){ }^{25} \mathrm{Mg}$ becomes fast enough to burn ${ }^{22} \mathrm{Ne}$ partially, and to be at the origin of a second neutron burst. This second neutron irradiation takes place during a relatively short period of time (several years), but gives rise to much higher neutron densities as compared to the first neutron irradiation during the interpulse. In cold AGB stars for which $T^{\text {bot }}<3.510^{8} \mathrm{~K}$, this second neutron burst is relatively inefficient and only reshapes the s-abundance distribution.

The s-process nucleosynthesis has also been studied in the framework of the rotationallyinduced mixing (Herwig et al. 2003; Siess et al. 2004). During the 3DUP stage, the extreme shear field that develops at the base of the convective envelope leads to the injection of protons into the adjacent ${ }^{12} \mathrm{C}$-rich layers. In contrast to the overshoot model, 
rotational mixing takes place on long timescales. For comparison, the diffusion coefficient at the base of the convective envelope is $D_{\text {rot }} \sim 10^{6} \mathrm{~cm}^{2} \mathrm{~s}^{-1}$ compared to the $10^{15} \mathrm{~cm}^{2} \mathrm{~s}^{-1}$ found in the overshoot model. But most importantly, the differential rotation that develops below the envelope during the 3DUP persists during most of the interpulse period. This on-going mixing leads to the contamination of the ${ }^{13} \mathrm{C}$-pocket with the ${ }^{14} \mathrm{~N}$ that forms on top of it and results in a strong reduction of the s-process efficiency. Although rotational mixing is an efficient mechanism to trigger the 3DUP and the partial mixing of protons, this mechanism rather inhibits the production of s-elements. The effects of metallicity need however further investigation.

The s-process models based on the partial mixing of protons at the time of the 3DUP and described so far have proven their ability to predict s-process abundance patterns consistent with many observations (see Sect. 5). However, some major problems still remain with this approach. In particular, the size of the partial mixing zone induced by convective overshooting - about $10^{-5} M_{\odot}$ - is one order of magnitude too small to explain the observed barium surface overabundances of up to 100 in AGB stars (Goriely \& Mowlavi 2000). In addition, it has been shown for about a decade now that most of the observations can be explained provided the s-process efficiency in the overshoottype model is modulated (e.g Busso et al. 2001). This modulation is usually obtained by modifying arbitrarily the ${ }^{13} \mathrm{C}$ content in the interpulse region. There is however no physical mechanisms that can be invoked at the present time to explain the large range of s-process efficiencies as indicated by the observations.

\subsection{The s-process in hot $A G B$ stars}

In intermediate-mass and/or low-metallicity AGB stars, the high temperatures that develop at the bottom of the convective zones, in the thermal pulse as well as in the envelope, can significantly modify the s-process nucleosynthesis described in the previous section.

In contrast with cool AGB stars, when the diffusive overshoot leads to the injection of protons into the adjacent ${ }^{12} \mathrm{C}$-rich layers, the ${ }^{12} \mathrm{C}(\mathrm{p}, \gamma){ }^{13} \mathrm{C}$ reaction can ignite before proton diffusion is ended and produces ${ }^{13} \mathrm{C}$ - and ${ }^{14} \mathrm{~N}$-rich layers that have time to carry on diffusing. Similarly to the rotational mixing case, the subsequent overlap of these ${ }^{14} \mathrm{~N}$-rich and ${ }^{13} \mathrm{C}$-rich layers strongly reduces the s-process efficiency. Depending on the thermodynamical conditions at the base of the convective envelope and on the depth of the extra-mixing (parameterized by the factor $f_{\text {over }}$, see e.g. Goriely \& Siess 2004 for the exact definition), the resulting s-abundance distribution can differ significantly, as shown in Fig. 4. A systematic analysis of the temperature at the base of the convective envelope at the time of the 3DUP provides a qualitative estimate of the metallicity and mass dependence of the efficiency of the overshoot s-process in AGB stars (Goriely \& Siess 2004). It appears that when the temperature in the mixing region is larger than $6 \times 10^{7} \mathrm{~K}$, no s-process nucleosynthesis occurs. At low temperatures (below $\sim 4 \times 10^{7} \mathrm{~K}$ ), a standard production of s-elements is expected and in the transition region, a large variety of efficiencies can be obtained (Fig. 4). However, our incapacity at the present time to estimate consistently the strength of the diffusive overshoot obscures our predictions concerning the interpulse s-process nucleosynthesis in these stars.

In addition to the complex above-described interplay between diffusion and nuclear processes, hot AGB stars can also be subject to a significant neutron irradiation within the convective thermal pulse due to the activation of the ${ }^{22} \mathrm{Ne}(\alpha, \mathrm{n}){ }^{25} \mathrm{Mg}$ reaction. Even in absence of the partial mixing of protons in the radiative C-rich layers, ${ }^{22} \mathrm{Ne}(\alpha, \mathrm{n}){ }^{25} \mathrm{Mg}$ can be responsible for a significant s-process. This convective s-process nucleosynthesis is known to be characterized by a short timescale (of the order of a few years) and strong $\left(N_{n} \simeq 10^{11}-10^{14} \mathrm{~cm}^{-3}\right)$ neutron densities. Like in massive stars, the convective 

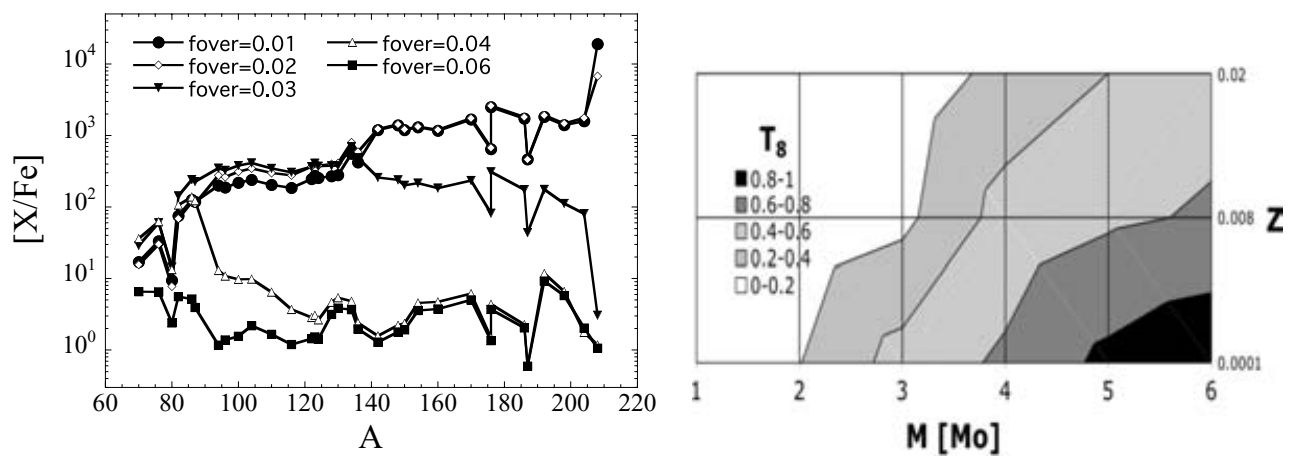

Figure 4. Left: Overproduction factors (with respect to solar) of s-only nuclei at the end of the interpulse phase in the partial mixing zone resulting from the diffusive overshoot with different values of the overshoot parameter $f_{\text {over }}$ characterizing the extent of the mixing zone. For more details, see Goriely \& Siess (2004). Right: Average temperature at the base of the convective envelope at the time of the 3DUP for a grid of AGB models of mass $M=1,2,3,4,5,6 M_{\odot}$ and metallicity $Z=0.02,0.008$ and 0.0001 . Radiative s-process nucleosynthesis is favored when $T_{8} \lesssim 0.4$

mixing also has the particularity to continually bring to the bottom of the thermal pulse (where the neutron density is the highest due to the high temperatures) some fresh Fe seed nuclei. The resulting nucleosynthesis is a delicate balance between production of heavy elements by neutron captures and the dilution effects pulse after pulse, and is consequently sensitive not only to the thermodynamical conditions in the thermal pulse, but also the pulse overlap and to the extent of the 3DUP. For these reasons, the convective s-process is still subject to major uncertainties associated with the description of mass loss and of the convective boundaries. To illustrate the efficiency of the convective s-process, we show in Fig. 5 the s-abundance distributions resulting from a series of identical thermal pulses (corresponding to the 9 th pulse in a $3 M_{\odot} \mathrm{Z}=0.0001$ model star) for which the temperature profiles has been scaled to reach different maximum values at the bottom of the thermal pulse.

Due to the short and strong character of the neutron burst, the s-abundance distribution in such a scenario presents some specific features. In particular, the light Sr, Y, Zr elements are usually largely produced with respect to the second peak elements (Ba,La,Ce). The high neutron densities and temperatures are also responsible for a larger contribution to the so-called sr-nuclei with respect to the radiative s-process (Sect. 4.1). This concerns in particular ${ }^{86} \mathrm{Kr},{ }^{87} \mathrm{Rb},{ }^{96} \mathrm{Zr},{ }^{115} \mathrm{Sn}$, but also some long-lived radio-isotopes of particular interest ${ }^{60} \mathrm{Fe}$ or ${ }^{182} \mathrm{Hf}$. This particularity can be at the origin of a significant s-production of r-dominant elements, as shown in Sect. 5.

\section{Comparison with observations}

The overshoot scenario has shown its ability to explain a possible origin of the observed s-process nucleosynthesis. In particular, this scenario agrees remarkably well with recent observations of low-metallicity Pb-stars (Van Eck et al. 2001). However, it fails to reproduce another class of s-process-rich low-metallicity stars that are depleted in $\mathrm{Pb}$ (e.g. Aoki et al. 2002, Van Eck et al. 2003). Strictly applied, it turns out that the s-process nucleosynthesis within the overshoot scenario is essentially a function of the stellar metallicity. However, observations clearly indicate that AGB stars of a given metallicity are subject to a significant modulation of the s-process efficiency. For the last decade, such a modulation has been achieved by modifying arbitrarily the amount of ${ }^{13} \mathrm{C}$ injected in 


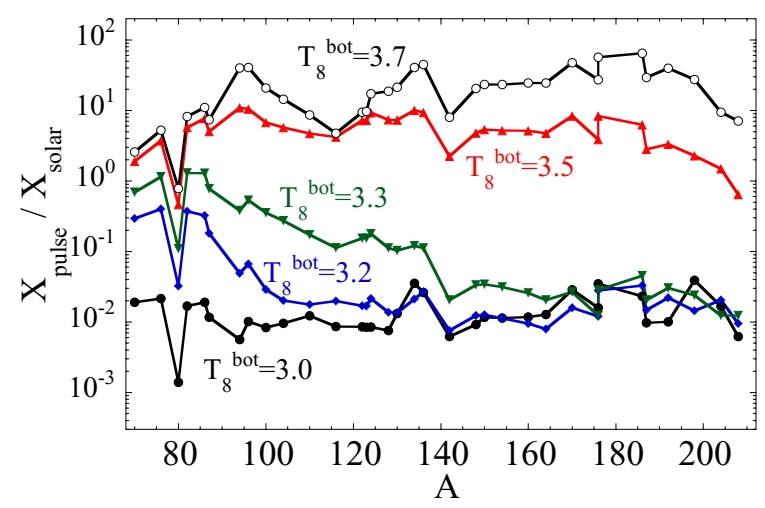

Figure 5. Overproduction factors (with respect to solar) of s-only nuclei at the end of the pulse phase resulting from the parametric convective s-process for different temperatures $T_{8}^{\text {bot }}$ (expressed in $10^{8} \mathrm{~K}$ ) at the base of the thermal pulse.

the C-rich layers (e.g Busso et al. 1999). As explained above, there is at the present time no physical mechanisms that can be invoked to justify such a wide range of conditions. Rotational effects can in principle provide such a modulation, but as shown by Siess et al. (2004) rotation is more likely to inhibit the production of neutrons. Note, however, that in this scenario, the role of the magnetic field has not been taken into account though it might be determinant. The strong differential rotation that develops at the base of the convective envelope could generate a magnetic field able to modify the rotational profile and hence the mixing as it does in massive stars (e.g. Maeder \& Meynet 2004). The interplay between diffusive mixing and H-burning described in Sect. 4.2 can also reduce the s-process efficiency, but in this case the decrease in the production of s-elements should be anti-correlated with the simultaneous effect of hot bottom burning, namely the production of ${ }^{14} \mathrm{~N}$ and ${ }^{13} \mathrm{C}$. No observation can confirm at the present time such an anti-correlation.

The convective s-process nucleosynthesis described in Sect. 4.2 also need to be confronted in detail with observations. Although such a scenario has been rejected on the basis of some specific observations, namely some observed $\mathrm{Mg}$ isotopic and $\mathrm{Sr} / \mathrm{Rb}$ abundance ratios (Busso et al. 2001), it is not clear to what extent this model could possibly explain the observed abundance distributions in specific low-metallicity stars. In contrast to the radiative s-process mentioned above, the s-process efficiency in the convective scenario increases with temperature. The production of s-elements is therefore correlated with hot bottom burning effects, and the surface s-elements indexes [hs/ls] or [vhs/ls] (where ls refers to the light s-process elements such as $\mathrm{Y}$ or $\mathrm{Zr}$, hs to the heavy Ba, La or Ce, and vhs to the superheavy ones like $\mathrm{Pb}$ ) should consequently be correlated with e.g. N. As explained in Sect. 4.2, the convective s-process can also account for a significant production of sr-nuclei. Recent observations (e.g Sivarani et al. 2004, Barbuy et al. 2005, Ivans et al. 2005) have revealed a non-negligible amount of very metal-poor stars strongly enriched in both s- and r-elements. The origin of such $\mathrm{r}+\mathrm{s}$ enhanced stars remains a mystery (see Barbuy et al. 2005 for a discussion of speculative sites). We show however in Fig. 6 that the radiative plus convective s-process in AGB stars can account for such observations. The radiative s-process during the interpulse phase gives rise to a s-abundance distribution similar to the one illustrated in Fig. 3, but the additional processing within the hot thermal pulse is held responsible for a large increase of the relative abundance of the so-called r-nuclei between Sm and Hf. Although this comparison 


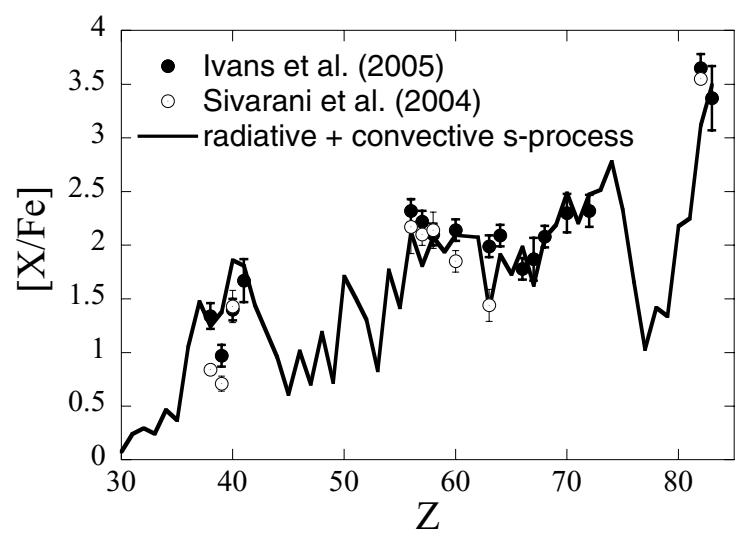

Figure 6. Comparison between the surface abundances of the metal-poor s+r enhanced star CS29497-030 with the prediction of the radiative plus convective s-process in massive AGB stars.

shows that the s-process in AGB stars can on its own explain such observations and consequently cast doubt on the very speculative scenarios often invoked, more observations are needed in particular to ascertain the r-process nature of the observed elements (e.g by the determination of abundances in the Pt region) before drawing any conclusions.

\section{Conclusions}

Although the classical site-independent s-process model has been very valuable in deriving the s- and r-contributions to the solar system, a special attention should be paid when applying such a solar decomposition to elements like $\mathrm{Pb}$, that can be produced by both the s- and r-processes, but also when interpreting the observed abundances in low-metallicity stars.

The s-process in massive stars might still require some specific investigations in particular concerning the effects of rotational mixing at low metallicity but most of all, it requires an improved determination of the ${ }^{22} \mathrm{Ne}(\alpha, \mathrm{n}){ }^{25} \mathrm{Mg}$ reaction rate.

AGB stars remain the major focus for s-process studies. Our ability to predict quantitatively and qualitatively the s-process nucleosynthesis in AGB stars remains highly dependent on stellar modeling. To improve the situation, progress must be made in our understanding of the convective boundaries where the extra mixing takes place. A better description of the internal transport processes must also be accompanied with a better knowledge of the mass loss which indirectly influences the thermodynamical conditions at the base of the convective envelope and consequently the 3DUP events. Improved models including both rotational and gravity-wave mixing in one- and multi-dimensional simulations would hopefully bring new insights in this direction (Arnett, this conference; Charbonnel, this conference). Although most of the efforts in the last decade have been devoted to the study of the s-process nucleosynthesis in low-mass (i.e cold) AGB stars, few has been done concerning hot AGB stars. This is regrettable since these stars show a very interesting nucleosynthesis due to the large temperatures found in their interiors. Clearly, they deserve additional investigation and these models should be further confronted with observations.

\section{Acknowledgements}

S.G. and L.S. are FNRS Research Associate. 


\section{References}

Angulo, C., Arnould, M., Rayet, M., et al. 1999, Nucl. Phys. A 656, 3

Aoki, W., Norris, J.E., Ryan, S.G., et al. 2002, ApJ 580, 1149

Arnould, M. \& Goriely, S. 2003, Phys. Rep. 384, 1

Barbuy, B., Spite, M., Spite, F., Hill, V., Cayrel, R., Plez, B., \& Petitjean, P. 2005, A\&A 429, 1031

Burbidge, E.M., Burbidge, G.R., Fowler, W.A., \& Hoyle F., 1957, Rev. Mod. Phys. 29, 547

Busso, M., Gallino, R., Lambert, D.L., Travaglio, C., \& Smith, V.V. 2001, ApJ, 557, 802

Busso, M., Gallino, R., \& Wasserburg, G.J. 1999, ARA\&A 37, 239

Chieffi, A., Dominguez, I., Limongi, M., \& Straniero, O. 2001, ApJ 554, 1159

Clayton, D.D., Fowler, W.A., Hull, T.E., \& Zimmerman, B.A., 1961, Ann. Phys. 12, 331

Denissenkov, P.A. \& Tout, C.A. 2003, MNRAS, 340, 722

Gallino, R., Arlandini, C., Busso, M. et al. 1998, ApJ 497, 388

Goriely,, S. 1999, A\&SA 342, 881

Goriely, S. \& Mowlavi, N. 2000, A\&SA 362, 599

Goriely, S. \& Siess, L. 2001, $A \& A$, 378, L25

Goriely, S. \& Siess, L. 2004, A\&A, 421, L25

Herwig, F., Blöcker, T., Schönberner, D., \& El Eid, M. 1997, A\& A, 324, L81

Herwig, F., Lugaro, M., \& Langer, N. 2003, ApJ, 593, 1056

Ivans, I., Sneden, C., Gallino, R., Cowan, J.J., \& Preston, G.W. 2005, ApJ in press

Käppeler, F., Beer, H., \& Wisshak, K. 1989, Rep. Prog. Phys. 52, 945

Langer, N., Heger, A., Wellstein, S., \& Herwig, F. 1999, A\&A 346, L37

Lugaro, M., Herwig, F., Lattanzio, J., Gallino, R., \& Straniero, O. 2003, ApJ, 586, 1305

Maeder, A. \& Meynet, G. 2004, A\&A 422, 225

Mazzali, P.A. \& Chugai, N.N. 1995, Astron. Astrophys. 303, 118

Prantzos, N., Hashimoto, M., \& Nomoto K., 1990 A\&A 234, 211

Rayet, M. \& Hashimoto, M. 2000 A $\& A 354,740$

Seeger, P.A., Fowler, W.A., \& Clayton, D.D. 1965, ApJS 11, 121

Siess, L., Livio, M., \& Lattanzio, J., 2002, ApJ, 570, 329

Siess, L., Goriely, S., \& Langer, N. 2004, A\& $A$ 415, 1089

Sivarani, T., Bonifacio, P., Molaro, P., Cayrel, R., Spite, M., Spite, et al. 2004, A $\& A$ 413, 1073

Straniero, O., Gallino, R., Busso, M. et al., 1995, ApJ 440, L85

Van Eck, S., Goriely, S., Jorissen, A., \& Plez, B. 2001, Nature 412, 793

Van Eck, S., Goriely, S., Jorissen, A., \& Plez, B. 2003, A\&SA 404, 291 12, 331 